\title{
Oxidative stress markers in severe preeclampsia and preeclampsia-related perinatal morbidity — preliminary report
}

\author{
Efser Oztas ${ }^{1}$, Sibel Ozler ${ }^{1}$, Aytekin Tokmak ${ }^{1}$, Ozcan Erel $^{2}$, Merve Ergin², \\ Dilek Uygur ${ }^{1}$, Nuri Danisman ${ }^{1}$ \\ 'Zekai Tahir Burak Women's Health Education and Research Hospital, Ankara, Turkey \\ ${ }^{2}$ Yildirim Beyazit University, Faculty of Medicine, Ankara, Turkey
}

\begin{abstract}
Objectives: The aim of the study was to determine maternal serum total antioxidant status (TAS), total oxidant status (TOS), oxidative stress index (OSI), paraoxonase (PON) and arylesterase levels in severe preeclamptic pregnants and also to investigate whether these parameters are implicated in the occurence of perinatal morbidity or not.

Material and methods: A case-control study was carried out including 60 pregnant women (30 with severe preeclampsia and 30 healthy controls). The optimal cut off points of oxidative stress markers for the diagnosis of severe preeclampsia and for the prediction of adverse perinatal outcomes were evaluated by receiver operating characteristic (ROC) analyses. Multivariate logistic regression analysis was used to determine if a relationship between adverse perinatal outcomes and serum oxidative stress markers was present or not.

Results: TAS (OR $=37.486,95 \% \mathrm{Cl} 3.535-397.519, \mathrm{p}=0.003)$, $\mathrm{TOS}(\mathrm{OR}=15.588,95 \% \mathrm{Cl} 2.135-113.818, \mathrm{p}=0.007)$ and arylesterase $(\mathrm{OR}=31.356,95 \% \mathrm{Cl} 2.284-430.548, \mathrm{p}=0.01)$ were found to be diagnostic for preeclampsia. Statistically significant positive correlation of adverse perinatal outcomes with serum TAS, PON and arylesterase levels were determined. Besides, a significant negative correlation was found between serum TAS levels and gestational week $(r=-0.342, p=0.007)$ and also between serum PON levels and birthweight $(r=-0.262, p=0.043)$.

Conclusions: Increased maternal serum TAS, TOS and arylesterase levels are significantly associated with the presence of severe preeclampsia. Furthermore, elevated maternal serum TAS, PON and arylesterase levels are significantly and positively correlated with adverse perinatal outcomes. We suggest that in preeclampsia increased oxidative status may cause adverse perinatal outcomes and antioxidants may be increased in order to protect the fetus against oxidative damage.
\end{abstract}

Key words: severe preeclampsia, perinatal outcome, oxidative stress markers

Ginekologia Polska 2016; 87, 6: 436-441

\section{INTRODUCTION}

Preeclampsia is a catastrophic, pregnancy-specific disorder, affecting $5-10 \%$ of all pregnancies and the leading cause of adverse pregnancy outcomes [1]. Diverse factors have been suggested to play a role in the etiopathogenesis of preeclampsia, including endothelial cell injury, impaired placentation and angiogenesis, changes in local oxygen tension, genetic predisposition, and immunological alterations [2-6]. Although the exact underlying mechanism is still obscure, it seems that maternal immune maladaptation is the triggering factor leading to inadequate placentation and altered endothelial function [7, 8]. Endothelial dysfunction results in increased systemic vascular resistance, leading to reduced perfusion in maternal systems, including the placenta [9]. Reduced perfusion aggravates placental ischemia-reperfusion injury, which is a strong stimulus for the production of reactive oxygen species (ROS) [10]. Additionally, increased generation of ROS, increased lipid peroxidation, and/or decreased antioxidant status further exacerbate endothelial dysfunction [11]. 
Increased oxidative stress has been already demonstrated to contribute in many cardiovascular diseases [12]. The characteristic vascular lesions in preeclampsia were shown to be similar to atherosclerotic plaques with lipid accumulation and infiltration of foam cells. Thus, it has been proposed that the changes in preeclampsia may be mediated by abnormal lipid peroxidation $[13,14]$. Lipid peroxidation causes cellular damage and vascular endothelial cell injury, and is used as an indicator of oxidative stress [15]. Oxidized LDL-cholesterol particles were also previously suggested to be involved in vascular endothelial injury in preeclampsia [16].

Paraoxonase (PON), is an antioxidant enzyme which is bound to HDL-cholesterol and has been shown to contribute in the prevention of LDL and HDL peroxidation [17]. PON has both, paraoxonase and arylesterase activity since it hydrolyzes organophosphates and aromatic esters [18]. Paraoxonase activity and oxidative status have been already shown to be altered in endothelial dysfunction-related disorders such as diabetes and cardiovascular diseases [19,20]. Similarly, one of the major and probably the causal problems in preeclampsia is endothelial dysfunction, so it may be proper to suggest an imbalance in oxidant and antioxidant status in the pathophysiology of preeclampsia. However, previous studies reported conflicting results of maternal serum PON and arylesterase levels in preeclampsia [15, 21]. Furthermore, the reported total oxidant status (TOS), total antioxidant status (TAS), and oxidative stress index (OSI) values were also controversial in different studies [10, 22]. Considering the results of the above-mentioned studies, we hypothesized that since preeclampsia and atherosclerotic diseases share a common pathophysiological mechanism, namely endothelial dysfunction, it may cause alterations in the perfusion of the utero-placental-fetal unit and lead to adverse perinatal outcomes.

\section{OBJECTIVES}

In our study, we aimed to determine maternal serum TAS, TOS, OSI, PON and arylesterase levels in severely preeclamptic pregnant patients and also to investigate whether these parameters were implicated in the occurrence of preeclampsia-related perinatal morbidity.

\section{MATERIAL AND METHODS}

A prospective case-control study was carried out between September 2013 and January 2014. Thirty patients diagnosed with preeclampsia were consecutively recruited from the Obstetrics and Gynecology Department, Zekai Tahir Burak Women's Health Education and Research Hospital. Severe preeclampsia was defined as blood pressure of $\geq 160 \mathrm{~mm} \mathrm{Hg}$ systolic or $\geq 110 \mathrm{mmHg}$ diastolic which occurs after 20 weeks of gestation in previously normoten- sive women, with proteinuria as urinary excretion of $\geq 0.3 \mathrm{~g}$ protein in a 24-hour urine specimen. In the absence of proteinuria, preeclampsia was defined as hypertension associated with at least one of the following: thrombocytopenia (platelet count of $<100,000 / \mu \mathrm{L}$ ), impaired liver function (increase in serum transaminases to twice the normal concentration), renal insufficiency (serum creatinine of $>1.1 \mathrm{mg} / \mathrm{dL}$ or doubling of serum creatinine in the absence of other renal diseases), pulmonary edema, and new-onset cerebral or visual disturbances [23]. The exclusion criteria were: multiple pregnancy, type I or II diabetes mellitus, chronic hypertension, hyper/hypothyroidism, chronic liver or renal disease, vascular and inflammatory diseases, smoking, and fetal anomaly. Thirty age- and BMI-matched healthy, pregnant women were recruited as controls.

All participants were evaluated at the initial admission. Clinical and anthropometric data were recorded. Gestational age was confirmed in all pregnant women by a routine ultrasonographic examination performed during the first trimester. Blood samples were obtained the time of diagnosis, following overnight fasting, and processed within $1 \mathrm{~h}$ after withdrawal by centrifugation, and stored at $-80^{\circ} \mathrm{C}$ until the day of the analysis. The adverse perinatal outcomes investigated in our study were as follows: low birthweight, preterm delivery, admission to a neonatal unit, and intrauterine growth retardation.

Serum TOS levels were determined spectrophotometrically using a method previously described by Erel, and the results were expressed in terms of micromolar hydrogen peroxide equivalent per liter $\left(\mu \mathrm{mol}_{2} \mathrm{O}_{2}\right.$ eqv./L) [24]. Serum TAS levels were determined using a novel method previously described by Erel, and the results were expressed as milimolar trolox equivalent per liter (mmol Trolox eqv./L) [25]. The ratio of TAS to TOS represents the OSI, an indicator of the degree of oxidative stress. Paraoxonase and arylesterase activities were measured using commercially available kits (RelassayR, Gaziantep, Turkey).

All participants provided a written informed consent. The study protocol was designed according to the principles of the Declaration of Helsinki and approved by the Local Ethics Committee.

BM SPSS Statistics for Windows, Version 20.0 (Armonk, NY: IBM Corp.) was used for statistical analysis. Continuous variables were tested for normality with the Kolmogorov-Smirnov test. Normally distributed data are presented as mean \pm standard deviations. Categorical comparisons were performed using the Chi-Square Test. For non-normally distributed data, median with data range (minimum to maximum) was used. We used the independent samples t-test and Mann-Whitney $U$ test for parametric and nonparametric groups, respectively. The optimal cut-off points of laboratory parameters determining preeclampsia 
were evaluated by ROC analyses calculating the area under the curve as giving the maximum sum of sensitivity and specificity for the significant test. Sensitivity and specificity were also calculated at the best cut-off point for each clinical measurement. In order to determine whether oxidative stress markers were independently associated with the presence of preeclampsia, multiple logistic regression analysis was used. Odds ratios and $95 \%$ confidence intervals for each independent variable were also calculated. The p-value of $<0.05$ was considered as statistically significant. Multivariate logistic regression analysis was also used to determine the presence of a relationship between adverse perinatal outcomes and serum oxidative stress markers. The p-value of $<0.05$ was considered as statistically significant.

\section{RESULTS}

Sixty pregnant women were enrolled in the study: 30 preeclamptic patients and 30 age- and BMI-matched healthy pregnant controls. The baseline characteristics of preeclamptic patients and controls are presented in Table 1. There were no statistically significant differences with respect to age, BMI, weight gain in pregnancy and gravidity between the groups. The percentage of adverse fetal outcomes and cesarean sections was significantly higher in the group with preeclampsia. Maternal serum TAS, TOS, PON, arylesterase levels and OSI were significantly higher in the preeclamptic group as compared to controls (Table 1).

Serum TAS, TOS, PON, arylesterase and OSI levels were again evaluated with ROC analysis, cut-off levels were determined, and the areas under curve (AUC) were calculated (Figure 1). The AUC, best cut-off values, sensitivity and speci- ficity for distinguishing the groups for each parameter are presented in Table 2. All parameters except for OSI were found to be statistically significant. Multivariate logistic regression analysis was then used to determine the presence of a relationship between the groups and the defined cut-off levels of the laboratory parameters. TAS (OR $=37.486$, $95 \% \mathrm{Cl} 3.535-397.519, \mathrm{p}=0.003)$, TOS (OR $=15.588,95 \%$ $\mathrm{Cl} 2.135-113.818, \mathrm{p}=0.007)$ and arylesterase $(\mathrm{OR}=31.356$, $95 \% \mathrm{Cl} 2.284-430.548, p=0.01$ ) were found to be indicative of preeclampsia (Table 3). Also, according to the results of the logistic regression model comparing the risk factors in patients with preeclampsia, the diagnostic performance of TAS, TOS, PON and arylesterase together was estimated at $90 \%$.

Further analysis was also performed to determine the presence of a correlation between oxidative stress markers and different variables in all pregnant participants of the study (Table 4). Statistically significant positive correlations between adverse perinatal outcomes and serum TAS, PON and arylesterase levels were determined. Besides, a significant negative correlation was found between serum TAS levels and gestational week $(r=-0.342, p=0.007)$ and also between serum PON levels and birthweight $(r=-0.262$, $p=0.043$ ). Multivariate logistic regression analysis, used to determine the predictive value of defined cut-off levels of the laboratory parameters and adverse perinatal outcomes, failed to determine such an association ( $p>0.05)$.

\section{DISCUSSION}

In our prospective case-control study, the elevated levels of maternal serum TAS, TOS and arylesterase levels were

Table 1. Baseline characteristics and laboratory parameters of preeclamptic patients and controls

\begin{tabular}{|c|c|c|c|}
\hline & Preeclampsia group $(n=30)$ & Control group $(n=30)$ & P value* \\
\hline Age (years) & $31.6 \pm 5.4$ & $31.5 \pm 5.7$ & 0.982 \\
\hline BMI $\left[\mathrm{kg} / \mathrm{m}^{2}\right]$ & $30.4 \pm 4.2$ & $29.9 \pm 3.9$ & 0.588 \\
\hline Weight gain [kg] & $13.0 \pm 4.4$ & $12.1 \pm 6.3$ & 0.493 \\
\hline Gravidity (n) & $2.0 \pm 2.0$ & $3.0 \pm 1.0$ & 0.214 \\
\hline Gestational weeks & $34.2 \pm 3.6$ & $38.5 \pm 1.3$ & $<0.001$ \\
\hline Fetal weight [g] & $2173.7 \pm 965.9$ & $3167.3 \pm 493.3$ & $<0.001$ \\
\hline Adverse perinatal outcome [n (\%)] & $18(60 \%)$ & $0 / 30(100)$ & $<0.001$ \\
\hline $\begin{array}{l}\text { Route of delivery } \\
\text { Cesarean/vaginal [n (\%)] }\end{array}$ & $28(93.3) / 2(6.7)$ & $13(43.3) / 17(56.7)$ & $<0.001$ \\
\hline TAS $[\mathrm{mmol} / \mathrm{mL}]$ & $2.1 \pm 0.4$ & $1.6 \pm 0.2$ & $<0.001$ \\
\hline TOS & $9.5 \pm 5.1$ & $5.6 \pm 1.7$ & $<0.001$ \\
\hline PON & $244.9 \pm 83.6$ & $191.1 \pm 56.5$ & 0.005 \\
\hline ARES & $309.5 \pm 84.0$ & $216.8 \pm 49.4$ & $<0.001$ \\
\hline OSI & $4.6 \pm 2.5$ & $3.5 \pm 1.1$ & 0.027 \\
\hline
\end{tabular}

Independent T Test - Mann-Whitney U test (Exact) - Pearson Chi-Square Test (Exact) \& Fisher Exact Test (Exact). Data are presented as Mean \pm SD (standard deviation) $\left[{ }^{*}\right.$ median \pm IQR (inter quartile range); $\left.{ }^{* *} \mathrm{n}(\%)\right] ; \mathrm{BMI}$ — body mass index; OSI — oxidative stress index 
Table 2. Cut-off values for laboratory parameters of preeclamptic patients and controls

\begin{tabular}{|c|c|c|c|c|}
\hline & \multirow{3}{*}{ Cut-off value } & \multicolumn{2}{|c|}{ Group } & \multirow{3}{*}{ P value } \\
\hline & & Study $(n=30)$ & Control $(n=30)$ & \\
\hline & & n (\%) & $n(\%)$ & \\
\hline \multirow{2}{*}{ TAS } & $<1.7225 / 1.7225 \leq$ & $3(10) / 27(90)$ & $19(63.3) / 11(36.7)$ & \multirow{2}{*}{$<0.001$} \\
\hline & & $0.859 \pm 0.46^{* * *}$ & $(90.0 \%)^{*} /(63.3 \%)^{* *}$ & \\
\hline \multirow{2}{*}{ TOS } & $<6.95 / 6.95 \leq$ & $11(36.7) / 19(63.3)$ & $24(80) / 6(20)$ & \multirow{2}{*}{$<0.001$} \\
\hline & & $0.793 \pm 0.056^{* * *}$ & $(63.3 \%)^{*} /(80.0 \%)^{* *}$ & \\
\hline \multirow{2}{*}{ PON } & $<216 / 216 \leq$ & $12(40) / 18(60)$ & $22(73.3) / 8(26.7)$ & \multirow{2}{*}{0.007} \\
\hline & & $0.702 \pm 0.068^{* * *}$ & $(60.0 \%)^{*} /(73.3 \%)^{* *}$ & \\
\hline \multirow{2}{*}{ ARES } & $<299.15 / 299.15 \leq$ & $16(53.3) / 14(46.7)$ & $30(100) / 0(0)$ & \multirow{2}{*}{$<0.001$} \\
\hline & & $0.809 \pm 0.055^{* * *}$ & $(53.3 \%)^{*} /(100 \%)^{* *}$ & \\
\hline \multirow{2}{*}{ OSI } & $\leq 3.1395 / 3.1395<$ & $9(30) / 21(70)$ & $15(50) / 15(50)$ & \multirow{2}{*}{0.128} \\
\hline & & $0.614 \pm 0.073^{* * *}$ & $(70.0 \%)^{*} /(50.0 \%)^{* *}$ & \\
\hline
\end{tabular}

TAS — total antioxidant status; TOS — total oxidant status; PON — paraoxonase; OSI — oxidative stress index

Table 3. Logistic regression model comparing the risk factors in patients with preeclampsia

\begin{tabular}{|l|c|c|c|c|c|c|}
\hline & B & S.E. & Wald & P & OR & $95 \%$ CI \\
\hline TAS $\geq 1.7225$ & 3.624 & 1.205 & 9.049 & 0.003 & 37.486 & $3.535-397.519$ \\
\hline TOS $\geq 6.95$ & 2.747 & 1.014 & 7.331 & 0.007 & 15.588 & $2.135-113.818$ \\
\hline PON $\geq 216$ & 1.633 & 0.996 & 2.686 & 0.101 & 5.118 & $0.726-36.070$ \\
\hline ARES $\geq 292.2$ & 3.445 & 1.337 & 6.645 & 0.010 & 31.356 & $2.284-430.548$ \\
\hline Constant & -4.999 & 1.430 & 12.226 & 0.000 & 0.007 & \\
\hline
\end{tabular}

$\mathrm{OR}$ — odds ratio; $\mathrm{Cl}$ — confidence interval; TAS — total antioxidant status; TOS — total oxidant status; PON — paraoxonase; OSI — oxidative stress index

Table 4. Correlation analysis between oxidative stress markers and different variables in all participants

\begin{tabular}{|c|c|c|c|c|c|c|c|c|c|c|c|c|c|}
\hline & & Age & BMI & GW & Gravida & EFW & WG & TAS & TOS & PON & ARES & APO & OSI \\
\hline \multirow{2}{*}{ TAS } & $r$ & 0.016 & -0.022 & -0.342 & -0.181 & -0.251 & -0.024 & 1 & 0,344 & 0,128 & 0,528 & 0.403 & -0.005 \\
\hline & $p$ & 0.905 & 0.867 & 0.007 & 0.166 & 0.054 & 0.853 & & 0.007 & 0.329 & 0.000 & 0.001 & 0.971 \\
\hline \multirow{2}{*}{ TOS } & $r$ & 0.214 & 0.096 & -0.177 & 0.047 & -0.115 & -0.015 & 0.344 & 1 & 0.264 & 0.123 & 0.238 & 0.925 \\
\hline & $p$ & 0.100 & 0.467 & 0.177 & 0.720 & 0.381 & 0.911 & 0.007 & - & 0.041 & 0.348 & 0.067 & 0.000 \\
\hline \multirow{2}{*}{ PON } & $r$ & -0.026 & 0.033 & -0.173 & 0.011 & -0.262 & -0.081 & 0.128 & 0.264 & 1 & 0.302 & 0.276 & 0.258 \\
\hline & $p$ & 0.845 & 0.805 & 0.187 & 0.934 & 0.043 & 0.540 & 0.329 & 0.041 & - & 0.019 & 0.033 & 0.047 \\
\hline \multirow{2}{*}{ ARES } & $r$ & -0.063 & 0.148 & -0.235 & -0.144 & -0.224 & 0.062 & 0.528 & 0.123 & 0.302 & 1 & 0.257 & -0.015 \\
\hline & $p$ & 0.635 & 0.258 & 0.070 & 0.272 & 0.086 & 0.641 & 0.000 & 0.348 & 0.019 & - & 0.047 & 0.907 \\
\hline \multirow{2}{*}{ OSI } & $r$ & 0.237 & 0.124 & -0.050 & 0.155 & -0.014 & -0.012 & -0.005 & 0.925 & 0.258 & -0.015 & 0.095 & 1 \\
\hline & $p$ & 0.068 & 0.344 & 0.703 & 0.236 & 0.917 & 0.925 & 0.971 & 0.000 & 0.047 & 0.907 & 0.468 & - \\
\hline
\end{tabular}

$\mathrm{BMI}$ — body mass index; TAS — total antioxidant status; TOS — total oxidant status; PON — paraoxonase; OSI — oxidative stress index

found to be associated with severe preeclampsia. Additionally, elevated maternal serum TAS, PON and arylesterase levels were significantly and positively correlated with adverse perinatal outcomes.

Oxidative stress has been already suggested to play an important role in the pathogenesis of placenta-related diseases [26]. Various studies showed increased oxidant status in preeclamptic pregnancies and suggested that oxidative stress is a major contributor in preeclampsia-related endothelial dysfunction [10,22, 27]. Our findings are consistent with the results of previous studies as we found elevated levels of maternal serum TOS to be associated with severe 
preeclampsia. Although increased oxidative stress is already accepted to be implicated in preeclampsia, either as a pathogenetic mechanism or as a consequence, the controversy about the possible roles of antioxidant status and enzyme levels remains [28]. A number of studies reported decreased TAS levels as a reduction in antioxidant defense mechanisms in preeclampsia $[22,27,29]$. These studies are contradictory to our results since we found significantly increased levels ofTAS in severely preeclamptic patients. However, the findings of two studies are consistent with our results, as they reported significantly increased TAS levels in maternal serum and cord blood of the newborns in preeclampsia [10, 30]. Additionally, there are also conflicting data among serum PON and arylesterase levels in preeclampsia. Kumru et al., reported significantly lower levels of PON and arylesterase in severe preeclampsia [21]. Conversely, Sarandol et al., showed that serum PON and arylesterase activities were not different in both, mild and severely preeclamptic patients [31]. We found significantly increased levels of PON and arylesterase and a positive correlation between both antioxidant enzymes with adverse perinatal outcomes in severe preeclampsia. Similarly, Acıkgoz et al., reported increased PON activity in preeclampsia [32]. Furthermore, Altunhan et al., found increased PON activity in cord blood of the newborns born to preeclamptic mothers and suggested that increased cord blood TAS and PON levels indicate that the fetus is protected against oxidative damage [30]. Their results are consistent with our study, as we found a positive correlation between adverse perinatal outcomes and increased antioxidant mechanisms. Although regression analysis failed to show a significant relation, we suggest that protection against oxidative stress may be higher in preeclamptic mothers, with a probable adverse pregnancy outcome since a significant positive correlation was determined. Another explanation for the statistically insignificant results may be the small size of the groups.

The main limitation of our study was a relatively small size of the groups. Although there was an evident correlation between serum TAS, PON, arylesterase levels and adverse perinatal outcomes, it missed the level of statistical significance due to the small sample size. Thus, this study needs to be validated with larger cohorts.

\section{CONCLUSIONS}

Increased maternal serum TAS, TOS and arylesterase levels are significantly associated with the presence of severe preeclampsia. Furthermore, elevated maternal serum TAS, PON and arylesterase levels are significantly and positively correlated with adverse perinatal outcomes. As increased activity of TAS, PON and arylesterase shows the secondary activation of defense mechanisms, we suggest that in preeclampsia increased oxidative status may cause adverse perinatal outcomes and antioxidants may be increased in order to protect the fetus against the oxidative damage.

\section{REFERENCES}

1. Roberts JM, Cooper DW. Pathogenesis and genetics of pre-eclampsia. Lancet. 2001, 357, 53-56.

2. Chen M, Yuan Z, Shan K. Association of Apolipoprotein J gene 866C凶T polymorphism with preeclampsia and essential hypertension. Gynecol Obstet Invest. 2005, 60, 133-138.

3. Lenfant C. National Education Program Working Group on High Blood Pressure in Pregnancy. Working group report on high blood pressure in pregnancy. J Clin Hypertens. (Greenwich) 2001, 3, 75-88.

4. Roberts JM, Gammil HS. Preeclampsia: recent insights. Hypertension. 2005, 46, 1243-1249.

5. Stanczuk GA, Mccoy MJ, Hutchinson IV, [et al.]. The genetic predisposition to produce high levels of TGF-beta1 impacts on the severity of eclampsia/pre-eclampsia. Acta Obstet Gynecol Scand. 2007, 86, 903-908.

6. Laresgoiti-Servitje E, Gomez-Lopez N, Olson DM. An immunological insight into the origins of pre-eclampsia. Hum Reprod Update. 2010, $16,510-524$.

7. Roberts JM, Lain KY. Recent insights into the pathogenesis of pre-eclampsia. Placenta. 2002, 23, 359-372.

8. Sibai B, Dekker G, Kupferminc M. Pre-eclampsia. Lancet. 2005, 365, 785-799.

9. Brennan LJ, Morton JS, Davidge ST.Vascular dysfunction in preeclampsia. Microcirculation. 2014, 21, 4-14.

10. Mert I, Oruc AS, Yuksel S, [et al.]. Role of oxidative stress in preeclampsia and intrauterine growth restriction. J Obstet Gynaecol Res. 2012, 38, 658-664.

11. Patil SB, Kodliwadmath MV, Kodliwadmath M. Lipid peroxidation and antioxidant activity in complicated pregnancies. Clin Exp Obstet Gynecol. 2009, 36, 110-112.

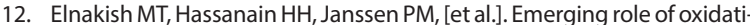
ve stress in metabolic syndrome and cardiovascular diseases: important role of Rac/NADPH oxidase. J Pathol. 2013, 231, 290-300.

13. Katabuchi $\mathrm{H}$, Yih $\mathrm{S}, \mathrm{Ohba} \mathrm{T}$, [et al.]. Characterization of macrophages in the decidual atherotic spiral artery with special reference to the cytology of foam cells. Med Electron Microsc. 2003, 36, 253-262.

14. Roberts JM. Endothelial dysfunction in preeclampsia. Semin Reprod Endocrinol. 1998, 16, 5-15.

15. Yaghmaei M, Hashemi M, Azarian A, [et al.]. Association of L55M and Q192R polymorphisms of paraoxonase-1 gene with preeclampsia. Arch Med Res. 2011, 42, 324-328.

16. Wakatsuki A, Ikenoue N, Okatani Y, [et al.]. Lipoprotein particles in preeclampsia: susceptibility to oxidative modification. Obstet Gynecol. 2000, 96, 55-59.

17. Aviram M, Rosenblat M, Bisgaier CL. Paraoxonase inhibits high-density lipoprotein oxidation and preserves its functions. A possible peroxidative role for paraoxonase. J Clin Invest. 1998, 101, 1581-1590.

18. Mackness MI, Arrol S, Abbot X, [et al.]. Protection of low-density lipoprotein against oxidative modification by high-density lipoprotein associated paraoxonase. Atherosclerosis. 1993, 104, 129-135.

19. Ayub A, Mackness MI, Arrol S, [et al.]. Serum paraoxonase after myocardial infarction. Arterioscler Thromb Vasc Biol. 1999, 19, 330-335.

20. Sozer V, Himmetoglu S, Korkmaz GG, [et al]. Paraoxonase, oxidized low density lipoprotein, monocyte chemoattractant protein-1 and adhesion molecules are associated with macrovascular complications in patients with type 2 diabetes mellitus. Minerva Med. 2014, 105, 237-244.

21. Kumru S, Aydin S, Gursu MF, [et al.]. Changes of serum paraoxonase (an HDL-cholesterol-associated lipophilic antioxidant) and arylesterase activities in severe preeclamptic women. Eur J Obstet Gynecol Reprod Biol. 2004, 114, 177-181.

22. Hilali N, Kocyigit A, Demir M, [et al.]. DNA damage and oxidative stress in patients with mild preeclampsia and offspring. Eur J Obstet Gynecol Reprod Biol. 2013, 170, 377-380.

23. Report of the American College of Obstetricians and Gynecologists'Task Force on Hypertension in Pregnancy. Hypertension in Pregnancy. Obstet Gynecol. 2013, 122, 1122-1131.

24. Erel O. A new automated colorimetric method for measuring total oxidant status. Clin Biochem. 2005, 38, 1103-1111.

25. Erel O. A novel automated direct measurement method for total antioxidant capacity using a new generation more stable ABTS radical cation. Clin Biochem. 2004; 37: 277-285. 
26. Jauniaux E, Poston L, Burton GJ. Placental-related diseases of pregnancy: Involvement of oxidative stress and implications in human evolution. Hum Reprod Update. 2006, 12, 747-755.

27. Yalcin S, Ulas T, Eren MA, [et al.]. Relationship between oxidative stress parameters and cystatin $C$ levels in patients with severe preeclampsia. Medicina. (Kaunas) 2013, 49, 118-123.

28. Llurba E, Gratacos E, Martin-Gallan P, [et al.]. A comprehensive study of oxidative stress and antioxidant status in preeclampsia and normal pregnancy. Free Radic Biol Med. 2004, 37, 557-570.

29. Karacay O, Sepici-Dincel A, Karcaaltincaba D, [et al.]. A quantitative evaluation of total antioxidant status and oxidative stress markers in preeclampsia and gestational diabetic patients in 24-36 weeks of gestation. Diabetes Res Clin Pract. 2010, 89, 231-238.

30. Altunhan $\mathrm{H}$, Annagur A, Kurban S, [et al.]. Total oxidant, antioxidant, and paraoxonase levels in babies born to pre-eclamptic mothers. $J$ Obstet Gynaecol Res. 2013, 39, 898-904.

31. Sarandol E, Safak O, Dirican M, [et al.]. Oxidizability of apolipoprotein B-containing lipoproteins and serum paraoxonase/arylesterase activities in preeclampsia. Clin Biochem. 2004, 37, 990-996.

32. Açıkgoz S, Bayar UO, Can M, [et al.]. Levels of oxidized LDL, estrogens, and progesterone in placenta tissues and serum paraoxonase activity in preeclampsia. Mediators Inflamm. 2013, 2013, 862-982. 